\title{
DEVELOPMENT CONCEPT OF GUARANTEED VERIFICATION ELECTRIC POWER SYSTEM SIMULATION TOOLS AND ITS REALIZATION
}

\author{
Alexander Gusev ${ }^{1}$, Aleksey Suvorov ${ }^{1, a}$ and Almaz Sulaymanov ${ }^{1}$ \\ ${ }^{1}$ Institute of Power Engineering, National Research Tomsk Polytechnic University, Lenina Avenue, 30, 634050, \\ Tomsk, Russia
}

\begin{abstract}
The analysis of existing problem reliability and verification of widespread electric power systems (EPS) simulation tools is presented in this article. Everything simulation tools are based on the using of numerical methods for ordinary differential equations. Described the concept of guaranteed verification EPS simulation tools and the structure of its realization are based using the Simulator having properties of continuous, without decomposition three-phase EPS simulation in real time and on an unlimited range with guaranteed accuracy. The information from the Simulator can be verified by using data only quasi-steady-state regime received from the SCADA and such Simulator can be applied as the standard model for verification any EPS simulation tools.
\end{abstract}

\section{Introduction}

As the basis for the solution most important tasks of design, research and exploitation of electric power systems (EPS) are used information about a full spectrum of various processes in power equipment and EPS as a whole, inevitable conclusion that the main reason for not enough reliable and effective solution to these problems are using false and incomplete information. At the same time the development and complexity EPS increases probability of system emergency. According to the summarized statistics $50 \%$ of such accidents in the world power industry are due to the wrong actions of relay protection, automation and the erroneous or delayed dispatching personnel actions $[1,2]$. The known specificity and complexity of modern EPS is excluded the possibility of obtaining the necessary information by full-scale test and by means of physical simulation. So, the main way of obtaining is mathematical simulation, its fullness and reliability is determined by two factors:

- the adequacy of the main equipment and EPS as a whole mathematical models;

- the tools ability carry out the solution of total EPS mathematical model on an unlimited range running quasi-steady-state and transient processes in real time with guaranteed acceptable accuracy and operativeness.

${ }^{a}$ Corresponding author : suvorovaa@tpu.ru 


\section{Theoretical substantiation of need simplifications and limitations using electric power systems numerical simulation}

In spite of the current level of physical and mathematical representation and description of the processes in the power equipment allows synthesizing adequacy mathematical models describing the full spectrum of the processes in all possible normal and abnormal regimes a power system. However, in this case the resulting total mathematical model of any real power system, even with the allowable simplifications, inevitably contains a stiff, non-linear and large size system of differential equations. Such system is bad stipulated for applicability conditions of numerical methods [3-5]. Such methods are basis for the computing core of all digital simulation EPS tools, without an exception. According to the theory of discretization techniques for ordinary differential equations, application of numerical methods is limited a set of conditions, the fulfillment of which determines the necessity used simplifications and limitations in digital simulation EPS tools:

- deep decomposition of continuous spectrum of normal and abnormal quasi-steady-state and transient processes in the EPS, according to such approach, conditionally allocated and individually calculated quasi-steady-state and transient processes, the last processes are divided into the electromagnetic and electromechanical stages, for each decompositions are used different and varying degrees simplified mathematical models and methods for their solution;

- considerable simplification the mathematical models of elements, especially the power network elements, static models in the form of the corresponding algebraic equations are presented such elements, that is principle unacceptable to the EPS simulation, three-phase circuits are replaced by single-phase;

- the interval of reproduction process is limited.

In addition, independently of these simplifications and limitations, local approximation error and the law of its transformation from step to step are not known. As a result a total methodological error is always unknown, and its definition is related to the category of fundamental challenges in the theory of discretization techniques for ordinary differential equations. In combination marked simplifications and limitations and total methodological error generate the problem fullness and reliability obtained information about a full spectrum of various processes in power equipment and power system as a whole by using digital EPS simulation tools. In this connection, verification of EPS simulation tools is an actual and required.

\section{Problem of verification electric power systems simulation tools and ways of its solving}

One of the first serious attempts at verification was carried out in the USA in connection with the analysis of a system emergency in the Western power system with more than $30 \mathrm{GW}$ of disconnecting the load [6]. The verification was performed by comparing the simulation results with the data of PMU, see figure 1. Obviously, the simulation results are completely satisfactory, but as you can see, there is a coincidence in the initial interval that corresponds to the theory of discretization techniques for ordinary differential equations, according to this, the methodological error of solution accumulates with increasing step-by-step calculations. Qualitative coincidences the simulation results and the field data have been achieved only by varying the parameters of the static characteristics simulated loads and field regulators, generator dead band. However, such attempt to verification uses "fit method" for a particular accident, and it is obvious that this "fit method" is not suitable for other accidents, it corresponds to the theory of discretization technique for ordinary differential equations. 


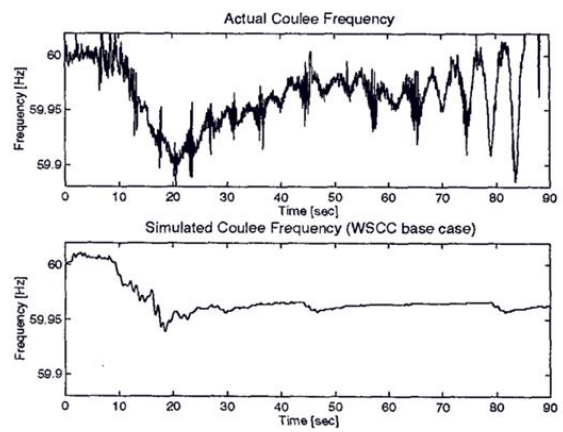

Figure 1. Grand Coulee unit 20 frequency - recorder and simulated using PMU data base

The verification task of digital simulations is also solved by «System Operator of the United Power System» in Russia. Using the similar technique and the published results are not solved the problem of guaranteed verification [7].

According to the analysis of existing approaches to the simulation tools verification is possible to achieve only quality results for a particular accident, essentially reflecting only the trend of changing parameters [7, 8]. Because the all digital simulation tools inevitably are used decomposition of processes, mathematical models and methods for solving are different for each decomposition, verification is not acceptable for one decomposition, and in connection with simplifications and uncertainties methodical error requires verification of the full continuous spectrum processes, but it is not realized in the near future.

\section{The alternative approach and the concept of solving the verification problem of electric power system simulation tools}

A radical way to deal with the simulation tools verification is used in developed concept:

1. The simulation results are compared to the standard model power system for guaranteed verification. The standard model power system has the properties of continuous reproduction quasisteady-state and transient processes with acceptable accuracy on an unlimited interval.

2. The standard model power system is used for adequate reproduction different operating modes: normal, emergency and post-emergency. Since the full continuum spectrum of normal and abnormal processes is reproduced as a result of methodically accurate solution on an unlimited interval the same highly adequate mathematical model, then each reproducible process, including quasi-steady-state mode at $50 \mathrm{~Hz}$, it is the result of this mathematical model solutions, and it is one of the single components of the spectrum of normal and abnormal processes. Since the highly adequate mathematical model is solved without decomposition and accurately, the experimental accuracy of reproduction quasi-steady-state mode can be extended to all significant frequency range $(0 \div 1000 \mathrm{~Hz})$, including switching overvoltage. The instrumental error integrated microelectronics is only limitation of continuously and methodically accurately reproduced spectrum of processes. It is determined by their amplitude-frequency and phase-frequency characteristics. This error is acceptable in a significant frequency range and the value of such error is $<1 \%$.

3. According to paragraph 2 , the verification of the standard model power system in all the important frequency range is possible by comparing the simulation results quasi-steady-state mode with the corresponding data of SCADA system.

4. For the reason that the verified significant frequency range is broad and the volume of data is enormous, the implementation of verification requires automation. Developed software and hardware tools for solving this problem. Tools are reproduced different scenarios of normal, emergency and post-emergency regimes, performed automatic comparison of the simulation results and presented the results of comparisons. 


\section{MATEC Web of Conferences}

The Hybrid Real Time Simulator of power systems (HRTSim) is developed in Institute of Power Engineering of Tomsk Polytechnic University. It represents multiprocessor software and hardware system that provide the real time continuous implicit integration of differential equations system describing processes in three phase power system. HRTSim allows avoiding decomposition of power system processes and simplification of power equipment models independently on the size of simulated EPS. Besides, the capability to provide any longitudinal and transversal commutations this system allows simulating any abnormal regime of power system [9, 10]. HRTSim has the following structure according to described concept, see Figure 2 and Figure 3. HRTSim industrial realization and implementation in the near future is very difficult, but it is a perspective task. However, the properties of the Simulator to fully meet all the requirements presented concept and it can be used as the standard model power system for guaranteed verification of EPS simulation tools.

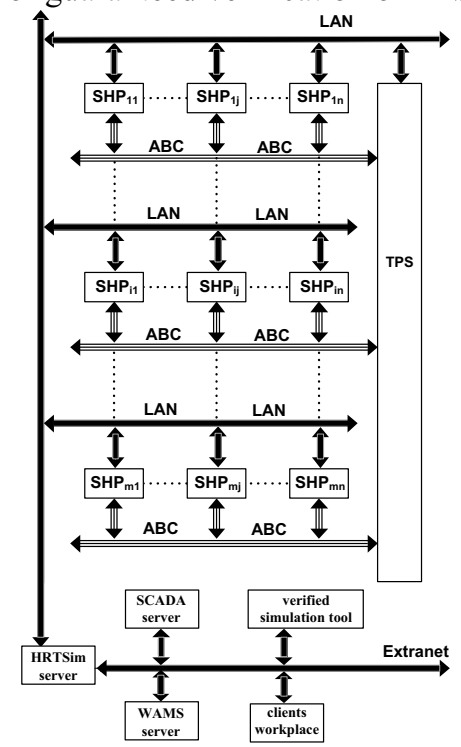

Figure 2. Structure of the HRTSim: SHP - specialized hybrid processors; LAN - local area network; TPS three-phase switch; ABC - three-phase input/output of SHP

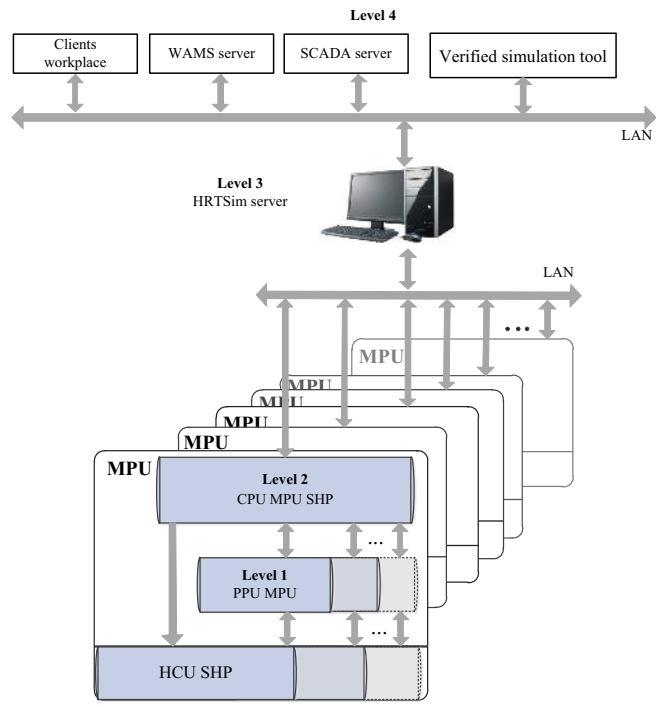

Figure 3. The structure of the multi-level information and control system: CPU - central processing unit, MPU microprocessor unit, PPU - peripheral processor unit, $\mathrm{HCU}$ - hybrid coprocessor unit 


\section{Conclusions}

According to the experimental results, the following conclusions can be made:

- theoretically impossible to obtain the full and reliable information about entire spectrum of quasisteady-state and transient processes by using digital EPS simulation tools, based on, without exception, the using numerical methods;

- existing approach to verification is fundamentally limited by applying in digital EPS simulation tools the inevitable simplifications, limitations and the absence, impossibility to accumulate all required field data for solving the problem of verification different operation modes of equipment and power system as a whole: normal, emergency and post-emergency;

- the developed concept of guarantee verification and its realization allows to solve the described problem by comparing the simulation results with the standard model, such model is guaranteed authenticity, reliability and verified by using only obtained by the SCADA data the quasi-steady-state regime.

This work was supported by mega-grant "Hybrid simulation and control of smart grids".

\section{References}

1. Yu. Borovikov, A. Prokhorov, M. Andreev, IFOST, 2, 609 (2012).

2. M. Negnevitsky, N. Tomin, D. Panasetsky, N. Voropai, V. Kurbatsky, U. Hager, C. Rehtanz, Power Systems Computation Conference, 30 (2014).

3. G. Hall, JM. Watt, Modern Numerical Methods for Ordinary Differential Equations (USA, 1976).

4. E. Hairer, G. Wanner, Solving ordinary differential equations: nonstiff problems (London, 1987).

5. R. Hamming, Numerical methods for scientists and engineers (New York, 1962).

6. D. Kosterev, C. Taylor, W. Mittelstadt, IEEE Transactions on Power Systems, 14, 3 (1999).

7. B. Ayuev, S. Kouzmin, Y. Kulikov, 20th Congress of World Energy Council, 35 (2007).

8. A.V. Pazderin, E.S. Kochneva, IEEE International Energy Conference, 386 (2014).

9. R. A. Ufa, V. A. Sulaymanova, A. S. Gusev, MATEC Web of Conferences, 19 (2014).

10. Y. S. Borovikov, A. S. Gusev, A. O. Sulaymanov, R. A. Ufa, IOP Conference Series: Materials Science and Engineering, 66 (2014). 